\section{Cerebrovascular} Diseases
Cerebrovasc Dis 2003;15:1-3

DOI: $10.1159 / 000067692$

\title{
Message from the Editors
}

The work of the Editorial and Advisory Board members in addition to all the Ad Hoc reviewers listed on the next pages is gratefully acknowledged by the editors.

As usual we are particularly grateful to those members who served for years on either the Editorial or Advisory Boards and will now be leaving them: M. Fisher, Worcester, Mass.; J. van Gijn, Utrecht; J.W. Norris, Toronto; F. Aichner, Innsbruck; K. Asplund, Umeå; W. Koroshetz, Boston, Mass.; E. Mori, Himeji; E.B. Ringelstein, Münster; N.G. Wahlgren, Stockholm. We welcome as Associate Editors as well as to the Editorial and Advisory Boards, respectively: W. Hacke, Heidelberg; G.A. Donnan, Melbourne; R. Baumgartner, Zürich; A.M. Buchan, Calgary; W.-D. Heiss, Köln; R. Hobson, Newark, N.J.; A. Algra, Utrecht; M. Brainin, Maria Gugging; H.-C. Diener, Essen; R.T. Higashida, San Francisco, Calif.; S.M. Oppenheimer, Princeton, N.J.; Y. Shinohara, Kanagawa; I. Skoog, Göteborg; G.K. Steinberg, Stanford, Calif.

As usual, from time to time the cover design will change beginning with this issue, but series will remain, such as the Stroke Issue and the Stroke Vignette suggestions and contributions are most welcome.

M. Hennerici, Mannheim

J. Bogousslavsky, Lausanne

\section{KARGER}

Fax +41613061234 E-Mail karger@karger.ch www.karger.com (c) 2003 S. Karger AG, Basel

Accessible online at: www.karger.com/ced 
F. Aichner, Linz, Austria

M. Alberts, Durham, N.C., USA

A.V. Alexandrov, Houston, Tex., USA

J.-M. Annoni, Genève, Switzerland

K. Asplund, Umea, Sweden

J. Bamford, Leeds, United Kingdom

M.P. Barnes, Newcastle upon Tyne, UK

H. Barnett, London, Ont., Canada

A. Barth, Bern, Switzerland

C. Bassetti, Zürich, Switzerland

J.S.P. van den Berg, Nijmegen,

The Netherlands

G. Besson, Grenoble, France

J. Biller, Indianapolis, Ind.,USA

S. Blecic, Brussels, Belgium

T. Brandt, München, Germany

T. Brandt, Heidelberg, Germany

J.M. de Bray, Angers, France

J.C.M. Brust, New York, N.Y., USA

M. Burnier, Lausanne, Switzerland

E. Camenzind, Genève, Switzerland

L. Candelise, Milano, Italy

A. Carolei, L'Aquila, Italy

A. Carota, Genève, Switzerland

P. Cesaro, Créteil, France

H. Chabriat, Paris, France

A. Chamorro, Barcelona, Spain

A. Cohen, Paris, France

A. Croquelois, Lausanne,

Switzerland

L. Csiba, Debrecen, Hungary

A. Culebras, New York, N.Y., USA

A. Czlonkowska, Warsaw, Poland

R. Darioli, Lausanne, Switzerland

C.-E. Dempfle, Mannheim, Germany

D.W. Desmond, Brooklyn, N.Y., USA

G. Di Pasquale, Bentivoglio, Italy

M. Dieterich, Mainz, Germany

K. Diserens, La Sarraz, Switzerland

D.W. Droste, Münster, Germany

M. Duchosal, Lausanne, Switzerland
K. Fassbender, Mannheim, Germany

C. Fieschi, Roma, Italy

C. M. Fisher, Boston, Mass., USA

M. Fisher, Worcester, Mass., USA

H. Flor, Mannheim, Germany

G. de Freitas, Rio de Janeiro, Brazil

H.J. Freund, Düsseldorf, Germany

A. Gass, Mannheim, Germany

J. Ghika, Lausanne, Switzerland

J. van Gijn, Utrecht,

The Netherlands

M. Ginsberg, Miami, Fla., USA

L.B. Goldstein, Durham, N.C., USA

D.I. Graham, Glasgow, UK

A. Grau, Heidelberg, Germany

C. Groden, Mannheim, Germany

J. van der Grond, Utrecht, The Netherlands

J. Grotta, Houston. Tex., USA

V. Hachinski, London, Ont., Canada

J. Harenberg, Mannheim, Germany

R.G. Hart, San Antonio, Tex., USA

D. Hayoz, Lausanne, Switzerland

C. Helgason, Chicago, Ill., USA

W. Hense, Münster, Germany

A. Hetzel, Freiburg, Germany

L. Hirt, Lausanne, Switzerland

H. Hof, Mannheim, Germany

A. Jaillard, Grenoble, France

P. Jallon, Genève, Switzerland

X. Jeanrenaud, Lausanne, Switzerland

T. Kanapanyotides, Lausanne, Switzerland

M. Kaste, Helsinki, Finland

R. Kay, Hong Kong, People's Republic of China

J. S. Kim, Seoul, South Korea

J. Klingelhöfer, Chemnitz, Germany

T. Klockgether, Bonn, Germany

I. J. Koralnik, Boston, Mass., USA

W. Koroshetz, Boston, Mass., USA

G. Krämer, Zürich, Switzerland

R. von Kummer, Dresden, Germany

W. Kuschinsky, Heidelberg,

Germany
O. Lanczik, Mannheim, Germany

B. Laurent, Saint-Etienne, France

J. Leclerc, Indianapolis, Ind., USA

B. Lemmer, Mannheim, Germany

D. Liepsch, München, Germany

N. Löhr, Mannheim, Germany

K.O. Lövblad, Genève, Switzerland

E. Lugaresi, Bologna, Italy

P.A. Lyrer, Basel, Switzerland

P. Maeder, Lausanne, Switzerland

H. Markus, London, UK

P. Marx, Berlin, Germany

S. Meairs, Mannheim, Germany

R.A. Meuli, Lausanne, Switzerland

P.R.A. Meylan, Lausanne, Switzerland

O. Mielke, Mannheim, Germany

$P$. de Moerloose, Genève, Switzerland

E. Mori, Himeji, Japan

T. Moulin, Besançon, France

M. Mumenthaler, Zürich, Switzerland

J.-P. Neau, Poitiers, France

A. Nicolaides, London, UK

K. Niederkorn, Graz, Austria

N. Nighoghossian, Lyon, France

J.W. Norris, Toronto, Canada

L. Pantoni, Firenze, Italy

H.-W. Pfister, München, Germany

C. Pierrot-Deseilligny, Paris, France

E.-W. Radü, Basel, Switzerland

L. Regli, Lausanne, Switzerland

M. Reichhart, Lausanne, Switzerland

J. de Reuck, Gent, Belgium

E.B. Ringelstein, Münster, Germany

A. H. Ropper, Boston, Mass., USA

J. Röther, Hamburg, Germany

R. L. Sacco, New York. N.Y., USA

W. Sandmann, Düsseldorf, Germany

K. Sartor, Heidelberg, Germany

P. Schmiedek, Mannheim, Germany

S. Schwab, Heidelberg, Germany 
M. Siebler, Düsseldorf, Germany

M.V. Singer, Mannheim, Germany

R. Stajzel, Genève, Switzerland

J. Stam, Amsterdam,

The Netherlands

F. Staub, Lausanne, Switzerland

W. Steinke, Düsseldorf, Germany

G. Stoll, Würzburg, Germany

F. Stögbauer, Münster, Germany

M. Sturzenegger, Bern, Switzerland

B.E. Tettenborn, St.Gallen,

Switzerland

L. Thommessen, Bergen, Norway
A. Thron, Aachen, Germany

E. Touzé, Paris, France

N. de Tribolet, Lausanne, Switzerland

A. Valavanis, Zürich, Switzerland

P. Vuadens, Sion, Switzerland

G. Waeber, Lausanne, Switzerland

N.G. Wahlgren, Stockholm, Sweden

J.M. Wardlaw, Edinburgh, UK

C. Weiller, Hamburg, Germany

C. Weimar, Essen, Germany

B. Widder, Günzburg, Germany
E.F.M. Wijdicks, Rochester, Minn., USA

M. Wintermark, Lausanne, Switzerland

J. Wöhrle, Mannheim, Germany

F.J. van der Woude, Mannheim, Germany

T. Yamaguchi, Osaka, Japan

Y. Yonekawa, Zürich, Switzerland

H. Zeumer, Hamburg, Germany 